\title{
A QUINCE AÑOS DEL LEVANTAMIENTO ZAPATISTA: ALCANCES Y RETOS DE UN MOVIMIENTO LOCAL EN LA EDAD $G L O B A L^{1}$
}

\section{FIFTEEN YEARS AFTER THE ZAPATISTA UPRISING: SUCCESSES AND CHALLENGES OF A LOCAL MOVEMENT IN THE GLOBAL AGE}

\section{Geoffrey Pleyers*}

\begin{abstract}
RESUMEN
Este artículo propone un balance del zapatismo considerado como un "movimiento de experiencia". Si bien su impacto en la política mexicana ha sido limitado, los zapatistas lograron construir espacios autónomos donde ponen en práctica formas alternativas de organización colectiva y de relaciones sociales. Desempeñaron un proceso de profunda transformación de sus comunidades, teniendo la dignidad y la autonomía como ejes centrales. Sin embargo, la voluntad de centrar el movimiento en sus raíces locales y de dedicar la mayoría de su energía a un proceso de transformación local no significa que los alcances y desafíos del zapatismo se limitan al nivel local. Los insurgentes consideran, al contrario, que desarrollar alternativas prácticas en el nivel local es la clave de un cambio global de mayor alcance. Por lo tanto, el movimiento zapatista conlleva dos tendencias distintas pero complementarias: la primera se dedica a la construcción de una autonomía local y al auto-gobierno de las comunidades, mientras la segunda busca ser un actor protagónico en la escena social y política nacional e internacional.
\end{abstract}

PALABRAS CLAVE: MÉXICO * POBLACIÓN INDÍGENA * CONFLICTOS POLÍTICOS * ZAPATISMO * MOVIMIENTOS SOCIALES * SOBERANÍA * MOVIMIENTOS INDÍGENAS

\section{ABSTRACT}

This article proposes a balance of the Zapatista movement considered as a "movement of experience". While their impact on Mexican policies has been limited, the Zapatista have managed to build autonomous spaces where they implement alternative forms

$1 \quad$ El autor agradece a Raúl Ornelas y a Alejandra Aquino por sus comentarios y a Pablo Avilés Flores y Rebeca Ornelas por su ayuda en la revisión del presente texto.
Investigador del Fondo Belga de la Investigación Científica (FNRS), Universidad Católica de Lovaina (UCL). Investigador Asociado al Centro de Análisis e Intervención Sociológica (CADIS) y en el Centre for the Study of Global Governance (London School of Economics). Geoffrey.Pleyers@uclouvain.be 
of collective organizations and social relations. Their communities have undergone a process of deep change based on two core values of the Zapatista movement: dignity and autonomy. However, the will that the movement is based on strong local roots and on a process of local transformation, it does not mean that its challenges are limited to the local level. Their struggle is properly universal and global, but they consider the development of alternative practices at the local level to be the key element of any global change. The Zapatista movement relies thus on a complex combination between two distinct trends: one focuses on the building of local autonomy while the other remains very active on the national and international social and political arena.

KEYWORDS: MÉXICO * INDIGENOUS POPULATION * POLITICAL CONFLICTS * ZAPATISMO * SOCIAL MOVEMENTS * SOVEREIGNTY * INDIGENOUS MOVEMENTS

El primero de enero de 2009, el movimiento zapatista festejó el quinceavo aniversario de su insurgencia. Los indígenas rebeldes se levantaron para mejorar sus condiciones de vida $y$ transformar la relación entre los pueblos indios y el Estado. Se manifestaron en contra de la negación de su propia existencia, ya que los pueblos indígenas eran invisibles en el México que festejaba su "integración al primer mundo", como en aquel entonces el presidente de México Salinas de Gortari, calificó al inicio del Tratado de Libre Comercio de América del Norte. Los insurgentes se afirmaron como sujetos históricos y personales (Touraine, 2000) animados por la voluntad de tomar su destino entre sus manos, lo que, como indígenas chiapanecos, se les estaba negando a causa de cinco siglos de historia, del régimen político mexicano contemporáneo y de los proyectos de desarrollo económicos neoliberales.

\section{DIGNIDAD Y AUTONOMÍA: CAMBIO LOCAL, DESAFÍOS GLOBALES}

Construyeron sus reivindicaciones económicas, culturales, sociales, políticas y jurídicas alrededor de dos principios centrales: la dignidad (Le Bot, 1997: 192; Ceceña, 2000 y 2001) y la autonomía (Ornelas, 2004). La dignidad, definida como la afirmación de una humanidad común y la exigencia de ser respetado, es el corazón del movimiento zapatista ${ }^{2}$. Con su levantamiento,

2 Este valor aparece como central en muchos movimientos indígenas. Como lo expuso un delegado mapuche durante el Foro Social Mundial 2002, su los indígenas insurgentes afirmaron que "la dignidad humana no es sólo patrimonio de los que tienen resueltas sus condiciones elementales de vida, (...) también los que nada tienen de material poseen lo que nos hace diferentes de cosas y animales: la dignidad". (Comunicado del 13 de enero 1994, en Ejército Zapatista de Liberación Nacional (EZLN), 1994: 71).

Lo que pedimos y lo que necesitamos los pueblos indígenas no es un lugar grande ni un lugar chico, sino un lugar digno dentro de nuestra nación; un trato justo, un trato de iguales, ser parte fundamental de esta gran nación; ser ciudadanos con todos los derechos que merecemos como todos; que nos tomen en cuenta $y$ nos traten con respeto (Comandante David 16/03/2001, en Ceceña, 2001: 162).

Exigieron que esta dignidad y este respeto como seres humanos iguales $y$ diferentes, se transcribieran tanto en el derecho como en la actitud cotidiana de cada mexicano.

La autonomía constituye el otro pilar del zapatismo. Los indígenas insurgentes se levantaron contra la situación social mexicana en la que

... a partir de la concentración de poder, se ha establecido un control sobre los destinos de las comunidades, de los municipios, de lo local y de lo regional de manera que estos últimos niveles son despojados de cualquier fuerza, de cualquier autonomía para regir y orientar su vida colectiva (Zermeño, 2005: 127-128).

reivindicación mayor es clara: "Somos seres humanos y queremos ser considerados como tales". 
Los zapatistas consideran que la afirmación de su dignidad pasa por la reivindicación de un control sobre sus vidas y sobre las decisiones que les afectan pero que estaban tomadas por mandatarios políticos y económicos muy alejados de la vida de los indígenas de Chiapas (EZLN, 1994: 51-54). Por lo tanto, entre las mayores exigencias de los movimientos indígenas se encuentran la reapropiación de sus territorios y de sus recursos naturales y las exigencias de autonomía ${ }^{3}$ y de autodeterminación, que consideran como "la oportunidad de construirnos, dentro de este país, como una realidad diferente ${ }^{4}$ ". Se trata también de perpetuar algunos elementos de su modo de vida, costumbres y tradiciones ancestrales sin que ello signifique dejar de ser ciudadano de una nación más amplia en la cual sea reconocido su derecho a ser iguales y diferentes.

El hecho de colocar a la autonomía como el centro de su movimiento, no significa que sus alcances se limiten al nivel local. Lejos de limitarse a sus comunidades indígenas, dieron a su demanda por "Democracia, Libertad y Justicia" un alcance propiamente universal (EZLN, 1994: 243). El primero de enero de 1994 se levantaron por un México democrático y en contra del neoliberalismo, denunciando el sistema político mexicano y el Tratado de Libre Comercio de América del Norte. La Sexta Declaración de la Selva Lacandona de 2006 reitera fuertemente esta dimensión del movimiento, reafirmando la implicación del zapatismo en la lucha global en contra de la dominación del dinero. El desafío del zapatismo no puede limitarse a una transformación de los territorios rebeldes. Se centran en las comunidades locales por que consideran que un cambio global se construye desde lo local, el cual permite poner en práctica alternativas concretas en la vida cotidiana y en la organización comunitaria. Es a partir de allí que

3 De igual manera, la Coordinación Nacional de los Indígenas de Colombia exigió al Estado "el respeto y la garantía de los derechos legítimos a la autodeterminación cultural, social, política y económica, a sus tierras, a su cultura, a sus formas propias de organizarse y de desarrollarse así como a una educación conforme a sus intereses y necesidades" (Padilla, 2000: 220).

Marcos, entrevista citada en Pardo Pacheco, 2001: 139. buscan cambiar la vida de los indígenas y que se involucran en luchas nacionales y globales, como la oposición a la Organización Mundial del Comercio, la denuncia de las deficiencias del sistema político mexicano o la implicación de los zapatista con otros pueblos indios de México $y$ de las Américas (Hocquenghem, 2009).

\section{1994-2001: DIÁLOGO Y MARCHAS PARA EL RECONOCIMIENTO INSTITUCIONAL DE LA AUTONOMÍA}

Siguiendo el largo recorrido de la resistencia indígena, de su organización local y de la defensa de su cultura, prácticas y valores de los pueblos indios en varios Estados del Sur de la República Mexicana; los zapatistas se movilizaron para que los pueblos indígenas y su autonomía local fueran reconocidos por la ley y por las autoridades mexicanas. El levantamiento del primero de enero de 1994 y el éxito que encontró en ese momento en los medios de comunicación nacionales e internacionales, dieron una visibilidad sin precedente a esta causa colocando en la escena nacional, un debate que las autoridades políticas nacionales habían ocultado desde lustros. Los zapatistas negociaron con los representantes del Estado mexicano, propusieron reformas jurídicas y políticas y se comprometieron para la democratización del país. Además de los innumerables comunicados mediáticos (y muchas veces poéticos) del subcomandante Marcos y de marchas hacia la capital nacional, se convocaron en Chiapas varios encuentros con la sociedad civil mexicana e internacional con el objetivo de escuchar la opinión de los simpatizantes y de dar a conocer las perspectivas de los líderes zapatistas. Entre estos encuentros, destacan la Convención Nacional Democrática (1994) y el primer Encuentro Intergaláctico (1996). Amplias delegaciones extranjeras tomaron parte en cada una de estas reuniones y decenas de observadores extranjeros siguen pasando o quedándose algunas semanas en las comunidades desde hace más de diez años.

En 1995 y 1996, los zapatistas se sentaron en una larga negociación con una comisión federal. Llegaron a un acuerdo sobre el estatuto de las comunidades indígenas y el 
reconocimiento jurídico de los pueblos indígenas (Díaz Polanco y Sánchez, 2002). En el periodo que va desde la aprobación de los Acuerdos de San Andrés por las comunidades zapatistas en 1996 hasta el verano del 2001, los zapatistas invirtieron muchas de sus fuerzas para que estos acuerdos fueran ratificados por los legisladores en San Lázaro. Varias marchas salieron de la selva del sureste mexicano hacia la capital. Durante la primavera de 2001, más de un millón de simpatizantes de la causa zapatista se reunieron en el Zócalo de la Ciudad de México para recibir a los integrantes de la última $y$ más mediatizada de ellas: la "Marcha del Color de la Tierra" (Michel y Escrázaga, 2001). Una delegación zapatista fue recibida en el Congreso nacional donde la comandante Esther dirigió un mensaje fuerte a los legisladores.

Sin embargo, a pesar de numerosas iniciativas, el movimiento zapatista nunca alcanzó sus objetivos en el ámbito jurídico y de la política institucional. La reforma votada algunas semanas después de la marcha de 2001 no respondió a las esperanzas de los movimientos indígenas, ya que los legisladores se negaron a reconocer los pueblos indígenas como "sujetos de derecho".

\section{DESPUÉS DE 2003: LOS CARACOLES Y LA AUTONOMÍA LOCAL}

Con el rechazo de los legisladores mexicanos a reconocer los pueblos indígenas como sujetos de derecho y a dar un estatuto legal a la autonomía de las comunidades indígenas, se abrió una nueva etapa en la cual el movimiento dejó de intentar influir en los actores políticos mexicanos y se enfocó aún más en el desarrollo de la autonomía que las comunidades estaban gozando de facto desde el primero de enero de 1994.

Fuera del sistema partidario y de las instituciones mexicanas, los municipios autónomos zapatistas organizan la vida de varios pueblos y aldeas. Desde el 2003 existe un nivel más elevado de organización que agrupa a varios municipios autónomos, los cinco "Caracoles". Cada uno cuenta con su "Junta de Buen Gobierno" a cargo de la coordinación de los municipios, de las relaciones con el exterior y de la justicia. Cada junta cuenta con 15 y 25 delegados elegidos y tiene su forma particular de organización, según las necesidades de la región.

La ceremonia del "nacimiento de los caracoles", a la que acudieron miles de simpatizantes en agosto de 2003, marcó simbólicamente el inicio de esta nueva fase. Más que en las declaraciones políticas y a menudo poéticas, esta fase se centra en la construcción concreta $y$ a veces problemática de la autonomía local a través de las prácticas cotidianas. 'Mirar hacia lo social y la sociedad más que hacia el seno político y mediático'. Allí esta el mensaje inicial y central de "la otra campaña" que iniciaron los zapatistas a partir del verano de 2005. Un año antes de las elecciones presidenciales $y$ legislativas, cuando todo México - en particular la prensa, la televisión y los intelectuales- sólo tenían ojos para el terreno electoral, los zapatistas invitaron a sus simpatizantes a mirar hacia la sociedad, hacia los múltiples protagonistas que, en su modesto nivel, desarrollan alternativas locales al modelo neoliberal.

En la nueva etapa, a las marchas hacia la capital y a las grandes reuniones con la sociedad civil nacional e internacional organizadas entre 1995 y 2001, sucedieron los "Encuentros de los Pueblos Zapatistas con los Pueblos del Mundo" y las visitas de caravanas de simpatizantes mexicanos y extranjeros en las comunidades autónomas. Con estos encuentros, ya no se trata de influir en los responsables políticos, sino de fortalecer el proceso de autonomía local $y$ de compartir las experiencias de las comunidades con activistas que apoyan a los zapatistas o que desarrollan experiencias de autonomía en otros contextos.

Del 30 de diciembre de 2006 al 2 de enero de 2007, tuvo lugar en Oventic el primer "Encuentro del pueblo zapatista con el pueblo del mundo". Seis mil indígenas, 232 "autoridades locales zapatistas" y 1300 activistas de distintos estados de México y de 47 países del mundo escucharon los testimonios respecto a la organización concreta de la autonomía local en las comunidades zapatistas. Del 20 al 29 de julio de 2007, un segundo "Encuentro con los pueblos del mundo" llevó una caravana a tres 
comunidades autónomas, donde se informaron de los proyectos y retos de estas tres Juntas de Buen Gobierno. La comunidad de La Garrucha hospedó el tercer encuentro, del 28 de diciembre de 2007 al $1^{\circ}$ de enero de 2008 organizado por y para las mujeres. Cada una de las mesas reunió delegados de los cinco caracoles y se dedicó a un aspecto particular de la autonomía: los gobiernos locales, la educación, la salud, la ecología, la cultura, la economía, el trabajo colectivo o la lucha de las mujeres.

\section{LA CONSTRUCCIÓN DE UNA AUTONOMÍA LOCAL}

Aunque no se les ha reconocido en la ley mexicana, las comunidades zapatistas gozan de una autonomía de facto desde su levantamiento en 1994. Se convirtieron en "espacios de experiencias" (Pleyers, 2009; McDonald, 2006) donde se prueban prácticas organizativas alternativas y relaciones sociales distintas a las de la sociedad dominante. Los activistas buscan construir lugares distanciados de la sociedad capitalista que permiten a los actores vivir de acuerdo con sus propios principios, entablar relaciones sociales diferentes $y$ a partir de estas situaciones ejemplares, de cambiar las relaciones de poder $y$ los valores hacía una transformación más global: "Se trata de lograr construir la antesala del mundo nuevo, un espacio donde, con igualdad de derechos y obligaciones, las distintas fuerzas políticas se ‘disputen' el apoyo de la mayoría de la sociedad”. (Marcos en 1995 citado por Ornelas, 2004). Esta forma de pensar el cambio social radical se distingue de la idea clásica de la revolución, no por el radicalismo del cambio, sino por la manera de lograrlo (Holloway, 2002). No se trata de imponer un poder progresista desde arriba, pero de iniciar por prácticas alternativas concretas y ejemplares desde abajo. La autonomía de las comunidades es un elemento clave que permite crear estos "espacios de experiencia".

Los indígenas que compartían sus experiencias durante los "encuentros con los pueblos del mundo" consideraban la autonomía como un proceso que "permite al pueblo decidir cómo quiere vivir y cómo quiere organizarse a nivel político y económico": "La autonomía, es que nos gobernemos como pueblo indígena, que decidamos cómo queremos que trabajen nuestras autoridades sin depender de las políticas que vienen de arriba”. Sin embargo, como lo destacaba el comandante Brus Li, "no hay ninguna regla que nos diga cómo nos podríamos organizar para ser autónomos". La autonomía zapatista se construye paulatinamente, en la experiencia colectiva de resistencia $y$ de construcción de alternativas, $y$ no se basa en un razonamiento teórico o únicamente en un balance de las experiencias históricas (Ornelas, 2004), lo que la distingue radicalmente de los movimientos revolucionarios y de las guerrillas del siglo XX.

La organización de la vida cotidiana y de las autoridades políticas locales, según modalidades distintas del caudillismo dominante en Chiapas antes de 1994, es un proceso largo. Se trata de reorganizar las comunidades para que los delegados elegidos por los habitantes contribuyan a organizar la comunidad sin concentrar el poder, para que "manden obedeciendo". Para evitar que se constituya un grupo de mandatarios separado de la población, los cargos no duran más de tres años y no son reelegibles. Todos los habitantes de la comunidad asumen entonces un cargo comunitario varias veces en su vida.

La autonomía local de las comunidades zapatistas no se identifica con el retorno a una organización tradicional. Al contrario, busca cambios profundos, especialmente en lo que se refiere a las mujeres. Si bien los comandantes zapatistas reconocen que a veces continúan actitudes machistas, la situación y la auto-estima de las mujeres indígenas cambió mucho desde que la promoción de la igualdad de géneros en las comunidades se volvió un eje central de la lucha zapatista hace 15 años (EZLN, 1994: 107-110; Hernández Castillo, 1998). Antes de 1994, la situación de las mujeres era poco envidiable en algunas de las comunidades indígenas.

- Antes era muy difícil para nosotras, porque nadie nos tomaba en cuenta $y$ porque no teníamos el derecho de opinar ni de tomar decisiones sobre nuestra propia 
vida. Muchas tuvieron que casarse sin poder elegir sus maridos $y$ tuvieron después que aguantar golpes y humillaciones de sus maridos (Magdalena, primer Encuentro con los pueblos del mundo).

- Según lo que pensaban nuestros padres, abuelos y esposos, nosotras teníamos que aguantar todo y permanecer calladas (Elena, primer Encuentro con los pueblos del mundo).

Durante muchos años, el apoyo de las indígenas al movimiento permaneció muy discreto:

— escuchábamos y dábamos comida.

Poco a poco, muchas tomaron confianza $y$ se comprometieron en cargos importantes para la comunidad. De hecho, los participantes en cada uno de los tres "encuentros con los pueblos del mundo" quedaron impactados por la fuerza de las palabras de las decenas de mujeres zapatistas que dieron testimonio de su lucha por las mujeres, pero también por la educación, la salud y la organización autónoma.

El sector de la educación también evolucionó mucho estos últimos 15 años. Se construyeron escuelas nuevas (más de cincuenta en el caracol de Oventic). Escuelas primarias ya funcionan en todos los municipios zapatistas y el nivel secundario ya está funcionando en muchas zonas. Miles de mujeres adultas aprendieron a leer y a escribir. Rechazaron los maestros oficiales $y$ formaron sus propios maestros. Pensaron la educación autónoma como una alternativa al "individualismo promovido por las escuelas del gobierno" 5 . Impartir una parte de los cursos en sus lenguas cambió también la relación en las aulas. Se basaron en pedagogías alternativas e innovaciones educativas "culturalmente pertinentes" (Gutiérrez Narváez, 2006), como el método Freire, y en valores de la cultura indígena. El aprendizaje se hace de manera lúdica y participativa, e incluye el trabajo colectivo en el campo, ya

Un maestro zapatista, Primer encuentro de los pueblos zapatistas con los pueblos del mundo, 2007. que los zapatistas no quieren desconectar la enseñanza de la vida en las comunidades. Se aprende el español pero también el idioma indígena local, ya que "a través de ella se transmite mucho de la cultura y de los valores" que el movimiento zapatista busca rescatar. Por lo tanto, el programa de enseñanza zapatista no corresponde a los programas oficiales nacionales $y$ no tiene por objeto permitir el acceso de los alumnos a la educación superior o universitaria en las ciudades vecinas. Los zapatistas insisten en que "los jóvenes aporten sus competencias a sus comunidades".

La aplicación concreta de la autonomía local resulta una marcha larga y difícil. Trasladar los valores de igualdad y los ideales de autogestión a la práctica, continúa siendo un reto a cada instante. La gestión de las relaciones de poder y de las divergencias de opinión en las comunidades, la distribución equitativa de las tareas $y$ los debates en asambleas para alcanzar un consenso requiere un largo proceso de aprendizaje práctico y político. La autonomía parece más difícil aún al nivel económico. La vida sigue siendo difícil en estas regiones pobres y en la que se encuentran miles de refugiados desde hace más de diez años. Muchos municipios no son viables económicamente ya que no tienen suficiente tierra disponible para el cultivo, tanto por el gran número de desplazados como por la presencia de campamentos militares del ejército nacional. Estas regiones han dependido mucho de la ayuda de organizaciones internacionales, como "Médicos del Mundo" o de los Comités de Apoyo internacionales. Pero el apoyo de algunas ONG está disminuyendo con el tiempo. Sin embargo, debido a la crisis sin precedente que atraviesa el campo mexicano (Mestries, 2009) y a la permanencia del conflicto, las bases de una autonomía económica no han sido establecidas en las zonas zapatistas. ¿Cómo profundizar la democracia en una zona de conflicto donde el ejército insurgente es indispensable para proteger a los indígenas rebeldes de las agresiones militares y paramilitares? ¿Cómo lograr una sustentabilidad económica de las zonas rurales si muchas de las tierras siguen siendo ocupadas por el ejército mexicano, $y$ en un contexto de crisis estructural del campo mexicano desde hace más de 25 años? 
En los últimos años, los comités de apoyo nacionales e internacionales crearon varios circuitos alternativos de distribución para algunos productos de las comunidades zapatistas, especialmente el café y las artesanías. Ello asegura una retribución adecuada a algunas cooperativas de productores y artesanos. Este tipo de proyectos podrían volverse un elemento clave de la autonomía zapatista, ya que aseguran una base local de producción $y$ de ganancias sin entrar en contradicción con los valores y las luchas del movimiento. Sin embargo, el alcance de estos circuitos continúa siendo limitado y no bastan para establecer una base económica sustentable que logre mejorar el nivel de vida material de estas poblaciones, ya que está sometido a las mismas condiciones de crisis del campo que las otras regiones mexicanas. Por lo tanto, para muchos jóvenes, la migración aparece como la única opción para mejorar su nivel de vida (Aquino, 2009).

\section{DOS VERTIENTES DEL ZAPATISMO}

No se trata de idealizar las comunidades zapatistas. Como en cualquier grupo humano, pueden aparecer juegos de poder y existen divergencias de opinión. Se compensa en parte por la larga experiencia práctica de las asambleas, la cual a menudo ayuda a las comunidades a lograr un consenso entre los participantes. Por otra parte, en algunos aspectos, las actividades de los activistas entran en contradicción con el modelo de organización social demasiado horizontal que defienden en sus discursos. El EZLN tiene una organización militar y en consecuencia, muy vertical. El ejército zapatista es un elemento fundamental para defender el movimiento frente a la contra-insurgencia. No obstante, la comandancia y algunos grupos de la población se basan sobre otros referentes $y$ otra lógica de acción, y pueden divergir en algunos casos y originar fricciones. En su análisis de las reacciones de la comandancia frente a grupos de refugiados que quisieron recuperar las tierras que ocupaban antes del conflicto, S. Mélenotte (2009) estima que las autoridades locales zapatistas no siempre están atentas a las demandas de sus bases $y$ toman a veces decisiones que son motivadas menos por el bienestar de las poblaciones que por consideraciones estratégicas coherentes con su propia visión del movimiento: "A pesar de la creación de los caracoles, las autoridades municipales zapatistas siguen - por no decir "obedecen"- la línea y las instrucciones del Comité Clandestino Revolucionario Indígena y del EzLN" (Mélenotte, 2009). Sin embargo, conviene subrayar la dificultad de resolver estas contradicciones en un contexto muy tenso debido a la guerra de baja intensidad llevada por los paramilitares.

Los comunicados zapatistas se refieren poco a los retos de la construcción de la autonomía que experimentaban las comunidades locales. La mayoría de los comunicados se dedican a la situación política y social a nivel nacional, hasta tener un papel protagónico durante la campaña electoral. Durante las giras nacionales de la otra campaña, el subcomandante pasó varios meses sin regresar a Chiapas durante los cuales asumió posicionamientos en nombre del movimiento zapatista. También parece significativo que, mientras el subcomandante Marcos fue la figura clave de los encuentros mediáticos de la fase anterior, de las negociaciones con la COCOPA, de las marchas para el reconocimiento político de la autonomía y del posicionamiento del zapatismo en la arena política mexicana, casi no apareció en los "encuentros con los pueblos del mundo", los cuales se dedicaron a las experiencias cotidianas de la construcción de una autonomía local.

Desde su levantamiento, el zapatismo ha sido definido como la articulación de dos corrientes distintas. De un lado, los zapatistas centraron el proceso de cambio social en las experiencias alternativas de las comunidades. La construcción de la autonomía conllevaba al mejoramiento del nivel de vida de los indígenas. Por otro lado, desde su inicio, también se ha planteado el zapatismo como un actor del cambio a un nivel nacional y global, en favor de la democracia $y$ de la justicia, $y$ en contra del neoliberalismo y de la dominación del dinero. Estas dos corrientes no están disociadas, y en muchos aspectos son complementarias. Sin los vínculos 
y el apoyo nacionales e internacionales, no sería posible la defensa de la autonomía local. De igual manera, las comunidades locales siempre expresaron su apoyo incondicional a los líderes del EZLN con fuertes movilizaciones.

La segunda corriente erigió al movimiento zapatista como un actor político del debate nacional y como un componente del movimiento global de rechazo al neoliberalismo y a menudo, al capitalismo. Desde las primeras semanas del levantamiento, los zapatistas tomaron posición en la mayor parte de los grandes debates políticos y sociales mexicanos, denunciando el Plan Puebla-Panamá, los efectos de la política y de la ideología neoliberal, las condiciones de vida en las comunidades indígenas del país o la explotación de los recursos naturales por actores del capitalismo mexicano e internacional (EZLN, 1995). De hecho, M. Castells (1997) calificó a los zapatistas como la "primera guerrilla de la era de la información" por las grandes calidades de comunicación de su portavoz, el subcomandante Marcos, lo que permitió al movimiento insurgente alcanzar en seguida una presencia internacional, al punto de volverse la referencia mayor de la amplia corriente de la vía de la subjetividad del movimiento altermundialista en todo los continentes. Comités de apoyo a los zapatistas se crearon en decenas de ciudades europeas $y$ norteamericanas (Olesen, 2005) y muchos extranjeros pasaron algunas semanas en las comunidades como observadores del conflicto, lo que ha mantenido un contacto permanente entre los indígenas zapatistas y activistas extranjeros.

Sin embargo, los zapatistas siempre han desconfiado de las convergencias con otros movimientos, por miedo de diluir sus especificidades en un conjunto amplio $y$ de tener que renunciar al control total de sus actos $y$ de su comunicación, elemento central de su aura global. El largo proceso de deliberación zapatista basado en la participación activa de cada comunidad en las decisiones parece difícilmente aplicable dentro de un movimiento más vasto $y$ más heterogéneo (Pleyers, 2004b). De hecho, si muchos comunicados zapatistas se han referido a las luchas altermundialistas, los zapatistas buscaron preservar su autonomía frente a las movilizaciones, actores $y$ foros de este amplio movimiento. Sin embargo, el movimiento zapatista contribuyó de manera importante al éxito de este movimiento, particularmente por su cultura política y por su concepción del cambio que adoptaron muchos jóvenes activistas globales (Pleyers, 2004a; Juris y Pleyers, 2009). Al contrario de lo que había sido anunciado en varias ocasiones, los zapatistas nunca se juntaron en un evento altermundialistas y guardaron sus distancias con los Foros Sociales Mundiales. Su participación en las movilizaciones en contra de la cumbre de la Organización Mundial del Comercio en Cancún en septiembre de 2003 ha sido muy discreta $y$ limitada. Esto no quiere decir que las bases zapatistas no compartan la lucha en contra del neoliberalismo a nivel internacional, sino que, a partir del año 2001, les urgía más mejorar las condiciones de vida cotidiana y construir la autonomía en las comunidades. Intentaron entonces concentrar las fuerzas del movimiento en la organización de los municipios y en la defensa de los derechos de los pueblos indígenas.

De hecho, si bien el alcance internacional representa un éxito para el movimiento zapatista, su mayor fuerza, su "esencia" (EZLN, 1994: 133), permanece en las comunidades indígenas, quienes constituyen la base sobre la cual el movimiento ha podido construirse desde 1994, a pesar de las dificultades y de la guerra de baja intensidad llevada acabo en los territorios autónomos por el ejército y por grupos paramilitares. Los procesos de organización autónoma de las comunidades locales mostraron el vigor de un actor implicado en una transformación social, política y cultural anclada en las comunidades locales, de manera profunda $y$ a largo plazo. Cuando el posicionamiento político de Marcos pareció llegar a un punto sin salida en la tensa situación política mexicana, los "encuentros con los pueblos del mundo" de 2007 mostraron que la auto-organización local estaba saliendo adelante en los municipios zapatistas a pesar de las dificultades cotidianas $y$ de las contradicciones inherentes a un proceso basado en experimentaciones prácticas de las alternativas por los que la viven. 


\section{EL CAMBIO COMO PROCESO Y NO COMO RUPTURA}

Los zapatistas buscan producir ellos mismos sus formas de vida y afirmarse en su capacidad creadora contra las manipulaciones de las industrias culturales hegemónicas (Wieviorka, 2009: 40). Sostienen así una concepción del cambio social que no pasa tanto por influir sobre los responsables políticos como por la transformación respecto de la manera de vivir juntos a partir de alternativas concretas que pongan en práctica los valores del movimiento y una reafirmación de las formas de sociabilidad locales.

Para los zapatistas, el objetivo no precede a la acción, sino que le es concomitante. Este carácter performativo del compromiso ya había sido destacado por Gandhi, quien consideraba que: "Debemos encarnar el cambio que queremos ver en el mundo" (Memorial Gandhi, Mumbai, 2004). La lucha no es pues contra un enemigo solamente o un sistema externo, sino que también es con la personalidad de cada uno y en cada movimiento: "El primer cambio está adentro de cada uno". El principal reto del zapatismo se encuentra en la transformación de las relaciones sociales en el seno de las comunidades mismas, ya sea en las relaciones de producción, las decisiones políticas o en las relaciones entre géneros. El zapatismo conlleva también una profunda transformación en cuanto a la estima de los indígenas en sí mismos, de la que se hace eco el valor central de la dignidad.

En vez de una ruptura brusca y radical con la idea clásica de revolución que se dio a lo largo de la historia, el cambio social se concibe como un proceso. El "otro mundo posible" no surgirá mañana, luego de la "gran noche", sino que comienza aquí y ahora, en estos rincones intersticiales de la sociedad apropiados por los activistas y transformados en espacios de experiencia alternativos $y$ autónomos. El cambio no se limita a un nivel local, sino que se concibe de abajo hacia arriba (bottom-up): "No podemos cambiar el mundo si no empezamos por cambiarnos a nosotros mismos, a ayudar a nuestros vecinos, a ver lo que está pasando en nuestro barrio"6.

6

Un joven activista cercano a los zapatistas, Cancún, 2003.
Estos activistas consideran la lucha como un proceso de experimentación creati$v a$, por medio del cual se ponen en práctica los valores de un "mundo mejor". Buscan construir "otro mundo" a partir de sus prácticas y experiencias alternativas: "La rebelión debe ser una rebelión práctica, debe ser la construcción de otra manera de hacer, de otra sociabilidad, de otra forma de vida" (Holloway, 2003). Al rechazar los modelos y planes preconcebidos para crear el mundo mejor, los activistas de la vía de la subjetividad privilegian un aprendizaje a través de la experiencia por método de prueba y error en procesos de experimentación, ya que "se hace camino al andar" y que "se aprende a caminar caminando". En vez de luchar para tomar el poder, como lo han hecho los movimientos sociales de la sociedad industrial, en vez de adoptar prácticas de contrapoder, que tienen como objetivo contrarrestar los órganos de poder y la influenza de las grandes empresas, como lo sugería Montesquieu, los activistas de la subjetividad buscan crear espacios de experiencia libres de relaciones de poder y de dominación (Holloway, 2002: 65; Benasayag, Brand, et ál., 2001), fuera de la influencia de la ideología mercantil y de los comunitarismos.

El caso zapatista lleva también a subrayar la dimensión colectiva del proceso de cambio social. Las comunidades son actores mayores de este proceso y por lo tanto, están profundamente transformadas por él. El cambio profundo que constituyeron las nuevas relaciones entre mujeres y hombres en las comunidades zapatistas es tan solo una parte de los cambios internos en las comunidades, donde la auto-gestión se apoya en algunas herencias de la cultura indígena, pero también en el rechazo a otras tradiciones incompatibles con los ideales de "democracia, libertad y justicia”.

Si bien es necesario subrayar el potencial innovador de esta concepción del activismo $y$ del cambio social, también posee su cuota de ilusión y sus límites. El paso de un cambio individual y local a una transformación más global del sistema político y social sigue siendo el ángulo muerto de estos movimientos y 
de algunos teóricos que se suman a ellos ${ }^{7}$. La multiplicación de espacios limitados en los que se desarrollan prácticas alternativas no conduce necesariamente a un cambio global de la sociedad. Esta puede coexistir muy bien con un fortalecimiento de las políticas neoliberales en la sociedad o con un crecimiento del peso de los poderes económicos. Ahora bien, al desarrollar espacios al margen de la esfera política e institucional, ¿estos activistas no dan más prioridad a la «opción de salida» (Hirschman, 1973) que a una contestación en la arena social y política («voice») que podría contribuir a cuestionar esas políticas? Estos movimientos, al desplazar la lucha de la esfera política a la vida cotidiana, ¿no dejan el campo libre a sus adversarios, por ejemplo a nivel de la influencia en las instituciones o instancias de poder? Boron (2003) o Hardt y Negri (2000: 265) advierten sobre la idea según la cual «la batalla contra el Imperio podría ganarse por sustracción, renuncia o defección. Esta deserción (...) es la evacuación de los lugares de poder». La lógica de sustracción a los poderes políticos y económicos parece ser tanto menos sustentable cuanto que el paso de estos espacios a una escala más amplia continua siendo extremadamente vago.

\section{CONCLUSIÓN}

Quince años después del levantamiento, los zapatistas no han logrado transformar las leyes ni las instituciones nacionales y de la transición a la democracia a la cual contribuyó. Quedan más desencantos que esperanzas. Si el impacto político del zapatismo en la política institucional mexicana no ha estado a la altura de las movilizaciones durante los primeros años tras el levantamiento, sus alcances son considerables. Cambió profundamente las comunidades indígenas del sureste mexicano y mucho más allá, contribuyó a una transformación profunda de la auto-estima de los indígenas y de su posición en la sociedad mexicana e internacional. De invisibles, se volvieron actores importantes

7 Es, por ejemplo, el caso de J. Holloway (2003) o de M. Benasayag et ál. (2001). no sólo de México, sino de las Américas, tanto al sur como al norte del Río Grande (Le Bot, 2002 y 2009). Centrado su movimiento en la experiencia, la auto-organización y la transformación de las comunidades, el zapatismo logró articular reivindicaciones identitarias $y$ universales $^{8}$. Cuando defienden y afirman su cultura, su diferencia y sus valores, los zapatistas como muchos movimientos indígenas, expresan "un rechazo propiamente universal de la dominación de los mercados $y$ de la burocracia, $y$ defienden la autonomía de una manera de pensar, de vivir $y$ de comunicar que se articula $y$ se combina con otras maneras de pensar, vivir y comunicar" (Hocquenghem y Lapierre, 2002: 11).

Mientras muchos movimientos altermundialistas urbanos $\mathrm{u}$ occidentales que surgieron en la última década generaron una dinámica amplia pero de corto plazo, el proceso de transformación sigue vigente en las comunidades zapatistas quince años después del levantamiento. Al contrario de algunos movimientos altermundialistas que se quedaron en los discursos y en la construcción teórica de alternativas al neoliberalismo, los zapatistas experimentaron una organización autónoma y alternativa alrededor de valores antagónicos a la cultura comercial y competitiva. Como no lograron que sus demandas fueran reconocidas por los poderes Ejecutivo, Legislativo y Judicial mexicanos, consideraron que, "si no podemos cambiar el mundo, luchamos para que el mundo no nos cambie a nosotros $9 "$.

\section{BIBLIOGRAFÍA}

Albrow, M. The Global Age. Cambridge: Polity Press, 1996.

Aquino, A. "Chapitre 4. Entre le "rêve zapatiste" et le "rêve américain": la migration des

8 Esta combinación se revela mucho más compleja a un nivel político (Benhabib, 2002).

9 Beto, un delegado del Caracol nro. 4 en el primer encuentro de los pueblos zapatistas con los pueblos del mundo. 
jeunes zapatistes aux Etats-Unis”. Entre le mouvement social et l'expérience migratoire: Les enfants des luttes indiennes s'en vont au Nord. [Thèse de doctorat]. París: EHESS, 2009.

Benasayag, M.; Brand, U.; Gonzalez, H.; Holloway, J.; Mattini, L.; Negri, T. y Collectivo Situaciones. Contrapoder. Una introducción. Buenos Aires: De mano a mano, 2001.

Benhabib, S. The claims of cultures. Equality and diversity in the global era. Princeton: Princeton University Press, 2002.

Boron, A. "Poder, 'contrapoder' y 'antipoder'”. Chiapas 15. 2003: 143-162.

Castells, M. The information age 2: The Power of identity. Oxford: Blackwell, 1997.

Ceceña, A.E. Revuelta y territorialidad, América Latina, los nuevos actores sociales. Buenos Aires: Kohen \& Asociados Internacional, 2000.

Ceceña, A.E. "La marcha de la dignidad indígena". Sobre la marcha. Michel G. y Escárzaga F. (coord.). México. UAMRizoma, 2001: 161-178.

Díaz Polanco, H. y Sánchez, C. México diverso. México: Siglo XXI, 2002.

EZLn. Documentos y comunicados $1 \& 2$. México: Era, 1994 y 1995.

Gutiérrez Narváez, R. "Impactos del Zapatismo en la Escuela”. Liminar. Estudios Sociales y Humanísticos IV (1). 2006.

Hardt, M.; Negri, A. Empire. París: Exils, 2000.

Hernández Castillo, R.A. (coord.). La otra palabra: mujeres y violencia en Chiapas. México: CIESAS, 1998.
Hirschman, A. Exit, Voice, and Loyalty: Responses to Decline in Firms, Organizations and States. Boston: Harvard University Press, 1973.

Hocquenghem, J.; Lapierre, G. (coord.). Hommes de maïs, cours de braise. Cultures indiennes en rébellion au Mexique. París: L'insomniaque, 2002.

Hocquenghem, J. Le Rendez-vous de Vícam. Rencontre de peuples indiens d'Amérique. París: Rue des Cascades, 2009.

Holloway, J. Cambiar el mundo sin tomar el poder. Buenos Aires: Herramienta, 2002.

Holloway, J. "Anche un bacio può essere un movimento anticapitalista, intervista a John Holloway raccolta da Marco Calabria”. Carta. Febrero, 2003.

Juris, J. y Pleyers, G. "Alter-Activism: Emerging Cultures of Participation among Young Global Justice Activists". Journal of Youth Studies XII (1). 2009.

Le Bot, Y. El sueño zapatista. México: Plaza y Janez, 1997.

Le Bot, Y. (coord.). Indiens. Chiapas > Mexico $>$ Californie. Montpellier: Indigène Editions, 2002.

Le Bot, Y. La grande révolte indienne. Paris: Laffont, 2009.

McDonald, K. Global Movements. London: Blackwell, 2006.

Mélenotte, S. "Una experiencia zapatista: San Pedro Polhó, doce años después". Mestries F., Pleyers G. y Zermeño S. (coord.). Una nueva configuración de los movimientos sociales en México. Gobernanza democrática, fortalecimiento localregional $y$ actores culturales. Barcelona y México: Anthropos, 2009. 
Mestries, F. "Los movimientos sociales rurales en la década de la alternancia o las esperanzas frustradas". Mestries F., Pleyers G. y Zermeño S. (coord.). Una nueva configuración de los movimientos sociales en México. Gobernanza democrática, fortalecimiento localregional y actores culturales. Barcelona y México: Anthropos, 2009.

Mestries, F.; Pleyers, G. y Zermeño, S. (coord.). Nueva configuración de los movimientos sociales en México. Barcelona y México: Anthropos, 2009.

Michel, G.; Escrázaga, F. (coord.). Sobre la marcha. México: UAM-Rizoma, 2001.

Olesen, T. International Zapatismo. London: Zed Books, 2005.

Ornelas, Bernal R. "La autonomía como eje de la resistencia zapatista, del levantamiento armado al nacimiento de los caracoles". Ceceña A.E. (coord.). Hegemonías y emancipaciones en el siglo XXI. Buenos Aires. CLACSO, 2004: 133-172.

Padilla, G. "Droit fondamental indigène et droit constitutionnel". Alternatives Sud vII-2. 2000: 213-230.

Pardo Pacheco, R. "El movimiento zapatista de liberación nacional en la opinión pública”. Michel G. y Escrázaga F. (coord.). Sobre la marcha. México. UAMRizoma, 2001: 131-160.

Pleyers, G. «Des black blocks aux alteractivistes: Pôles et formes d'engagement des jeunes altermondialistes». Lien Social et Politiques 51. 2004a: 123-134.

Pleyers, G. "Social Forums as an ideal model of convergence". International Social Science Journal LVI (182). 2004b: 507-517.

Pleyers, G. "The World Social Forum: a Globalisation from below?". Societies without border III (1). 2008: 72-90.

Pleyers, G. Alter-Globalization. Becoming actor in the global age. Cambridge: Polity Press, 2009.

Touraine, A. ¿Podremos vivir juntos? Iguales $y$ diferentes. México: Fondo de Cultura Económica, 2000.

Wieviorka, M. (coord.). Otro mundo... Discrepancias, sorpresas y derivas en la antimundialización. México: Fondo de Cultura Económica, 2009.

Zermeño, S. La desmodernidad mexicana y las alternativas a la violencia y a la exclusión en nuestros dias. México: Océano, 2005. 